\title{
Prognostic value at 5 years of microvascular obstruction after acute myocardial infarction assessed by cardiovascular magnetic resonance
}

Gert Klug${ }^{1}$, Agnes Mayr², Sonja Schenk', Regina Esterhammer², Michael Schocke², Michael Nocker', Werner Jaschke ${ }^{2}$, Otmar Pachinger ${ }^{1}$ and Bernhard Metzler ${ }^{1 *}$

\begin{abstract}
Background: Early and late microvascular obstruction (MVO) assessed by cardiovascular magnetic resonance (CMR) are prognostic markers for short-term clinical endpoints after acute ST-elevation myocardial infarction (STEMI). However, there is a lack of studies with long-term follow-up periods (>24 months).

Methods: STEMI patients reperfused by primary angioplasty $(n=129)$ underwent MRI at a median of 2 days after the index event. Early MVO was determined on dynamic Gd first-pass images directly after the administration of $0.1 \mathrm{mmol} / \mathrm{kg}$ bodyweight Gd-based contrast agent. Furthermore, ejection fraction (EF, \%), left ventricular myocardial mass (LVMM) and total infarct size (\% of LVMM) were determined with CMR. Clinical follow-up was conducted after a median of 52 months. The primary endpoint was defined as a composite of death, myocardial re-infarction, stroke, repeat revascularization, recurrence of ischemic symptoms, atrial fibrillation, congestive heart failure and hospitalization.

Results: Follow-up was completed by 107 patients. 63 pre-defined events occurred during follow-up. Initially, 74 patients showed early MVO. Patients with early MVO had larger infarcts (mean: $24.9 \mathrm{~g}$ vs. $15.5 \mathrm{~g}, \mathrm{p}=0.002$ ) and a lower EF (mean: $39 \%$ vs. $46 \%, p=0.006$ ). The primary endpoint occurred in $66.2 \%$ of patients with MVO and in $42.4 \%$ of patients without MVO ( $<<0.05)$. The presence of early MVO was associated with a reduced event-free survival (log-rank $p<0.05$ ). Early MVO was identified as the strongest independent predictor for the occurrence of the primary endpoint in the multivariable Cox regression analysis adjusting for age, ejection fraction and infarct size (hazard ratio: $2.79,95 \%-\mathrm{Cl} 1.25-6.25, \mathrm{p}=0.012$ ).
\end{abstract}

Conclusion: Early MVO, as assessed by first-pass CMR, is an independent long-term prognosticator for morbidity after AMI.

Keywords: Microvascular obstruction, Cardiovascular outcome, Myocardial infarction, Cardiaovascular magnetic resonance

\section{Background}

Microvascular obstruction (MVO) after primary percutaneous coronary intervention (pPCI) for acute STelevation myocardial infarction (STEMI) is a no reflow phenomenon in coronary vessels smaller than $200 \mu \mathrm{m}$ despite adequate epicardial reperfusion [1]. Microvascular no-reflow can be caused by an interplay of multiple

\footnotetext{
* Correspondence: Bernhard.Metzler@uki.at

${ }^{1}$ Cardiology, University Clinic of Internal Medicine III, Medical University Innsbruck, Innsbruck, Austria

Full list of author information is available at the end of the article
}

factors, including embolization of thrombus and plaque, endothelial dysfunction, inflammation, myocardial edema and microvascular dysfunction after treatment of STEMI [2-5].

Classical measures of MVO are the thromobolysis in myocardial infarction (TIMI) myocardial perfusion grade [6] and myocardial blush grade [7] after successful epicardial perfusion as assessed by TIMI flow grade [8]. Myocardial perfusion grade and myocardial blush grade are associated with mortality after thrombolytic [6] and percutaneous [7] restoration of blood flow after myocardial infarction, independent of TIMI flow.

\section{Ciomed Central}


Cardovascular magnetic resonance (CMR) with its concepts of first-pass perfusion and late-Gd-enhancement (LGE) imaging is a unique tool for investigating $\mathrm{MVO}$ [9-12]. Early MVO is defined as a prolonged ( 60 sec) perfusion deficit in first-pass images [13]. Late MVO is usually assessed as an hypointense infarct core on LGE images acquired $10 \mathrm{~min}$ after contrast application [14]. Especially early MVO has been shown to correlate well with angiographic myocardial blush grade [15]. MVO can be studied by CMR at least up to 1 week after pPCI for STEMI [16].

The morphological type of coronary lesion, a large vessel diameter and a high thrombus load have an impact on the occurrence of slow- or no-reflow phenomenon after primary angioplasty [17]. Furthermore, MVO is predicted by the extent of the ischemic region and is associated with decreased baseline ejection fraction and wall motion scores[18]. We have previously shown the relation of cardiac troponin and $\mathrm{C}$-reactive protein to MVO after successful pPCI for STEMI [14]. Both early [19] and late [11,20] MVO have been shown to be significant and independent prognostic factors affecting clinical outcomes. So far, vasodilators [21,22], antiplatelet drugs [23] and thrombus aspiration [24] have been used with varying results [25]. The use of CMR in the detection of MVO offers new possibilities in the search for drugs to prevent MVO. Large multicenter trials now use CMR as the primary endpoint [26].

Despite the increasing awareness of the clinical importance of MVO, there is a lack of long-term followup ( $>24$ months) studies. Therefore, the aim of this study was to (a) assess clinical variables associated with early MVO and (b) evaluate the prognostic value of early MVO during a long-term follow-up (5 years).

\section{Methods}

\section{Patient population}

Patients reperfused by pPCI for STEMI at the University Hospital Innsbruck between 01/2005 and 12/2007 were asked to participate in the study. One-hundred and twenty-nine patients fulfilled inclusion criteria and gave their informed consent. The study was approved by the local ethics committee.

Inclusion criteria were (a) diagnosis of ST-elevation myocardial infarction (STEMI) according to the redefined ESC/ACC committee criteria [27] as first cardiac event (b) the exact determination of time from onset of symptoms until revascularization of infarct-related artery (pain-to-balloon-time), (c) Killip class $<2$ and no preexisting condition of heart failure. Furthermore, only patients with (d) no contraindications to CMR were eligible for this study.

Clinical follow-up was completed in 12/2010. The endpoints were defined by a consensus of the primarily involved investigators who were blinded to the CMR results. The primary endpoint was defined as a composite of death, myocardial re-infarction, stroke, repeat revascularization, recurrence of ischemic symptoms, atrial fibrillation, congestive heart failure and hospitalization of any cause. Secondary endpoints were defined as the individual endpoints of the composite primary endpoint.

Ischemic symptoms were defined as the presence of typical anginal pain. Congestive heart failure was defined as episodes of cardiac decompensation, including edemas or worsening dyspnea of at least NYHA class III-IV requiring medical attention. If more than one end-point occurred in one patient the earliest and/or most severe end-point was chosen.

\section{Biochemical measurements}

CK activity was determined by an enzymatic assay (Roche Diagnostics, Mannheim, Germany) and cTnT concentrations were measured by $4^{\text {th }}$ generation cTnT enzyme immunoassays (Roche Diagnostics, Mannheim, Germany) [28].

Blood samples for CK and cTnT measurements were collected routinely according to a standard protocol at least 3 times during the first 24 hours after admission and then daily. Maximum CK and cTnT levels as well as estimated cumulative $\mathrm{CK}$ and cTnT release were determined. Maximum cTnT and CK values served as an estimate of infarct size [29,30].

\section{Cardiovascular magnetic resonance}

CMR was performed within at most 8 days after STEMI during hospitalization. All studies were performed on a 1.5 Tesla (T) MR scanner (Magnetom Avanto, Siemens, Erlangen, Germany). The CMR protocol was described in detail previously [12] (Figure 1a).

End-diastolic volume (EDV) and end-systolic volume (ESV) as well as ejection fraction (EF) and myocardial mass (MM) were obtained from short axis cine-MR images, acquired using breath-hold, with retrospective ECG-triggered true-FISP. Evaluation was performed on standard software (ARGUS, Siemens) by an experienced reader (G.K.) blinded to the clinical data.

First-pass perfusion images were obtained in three short-axis sections centered on the mid-papillary muscle using an electrocardiograph-triggered T1-weighted inversion-recovery true-FISP sequence. A bolus injection of $0.1 \mathrm{mmol} / \mathrm{kg}$ body mass gadolinium contrast bolus (Spectris, Medrad, Pittsburgh, PA) was administered by using an infusion pump (Spectris, Medrad, Pittsburgh, $\mathrm{PA})$ at $5 \mathrm{ml} / \mathrm{sec}$ [31]. Sixty dynamic images were acquired simultaneously at each of the three sections during the first pass of the contrast agent within the myocardium. Registration of microvascular perfusion defects on first-pass perfusion images was performed 

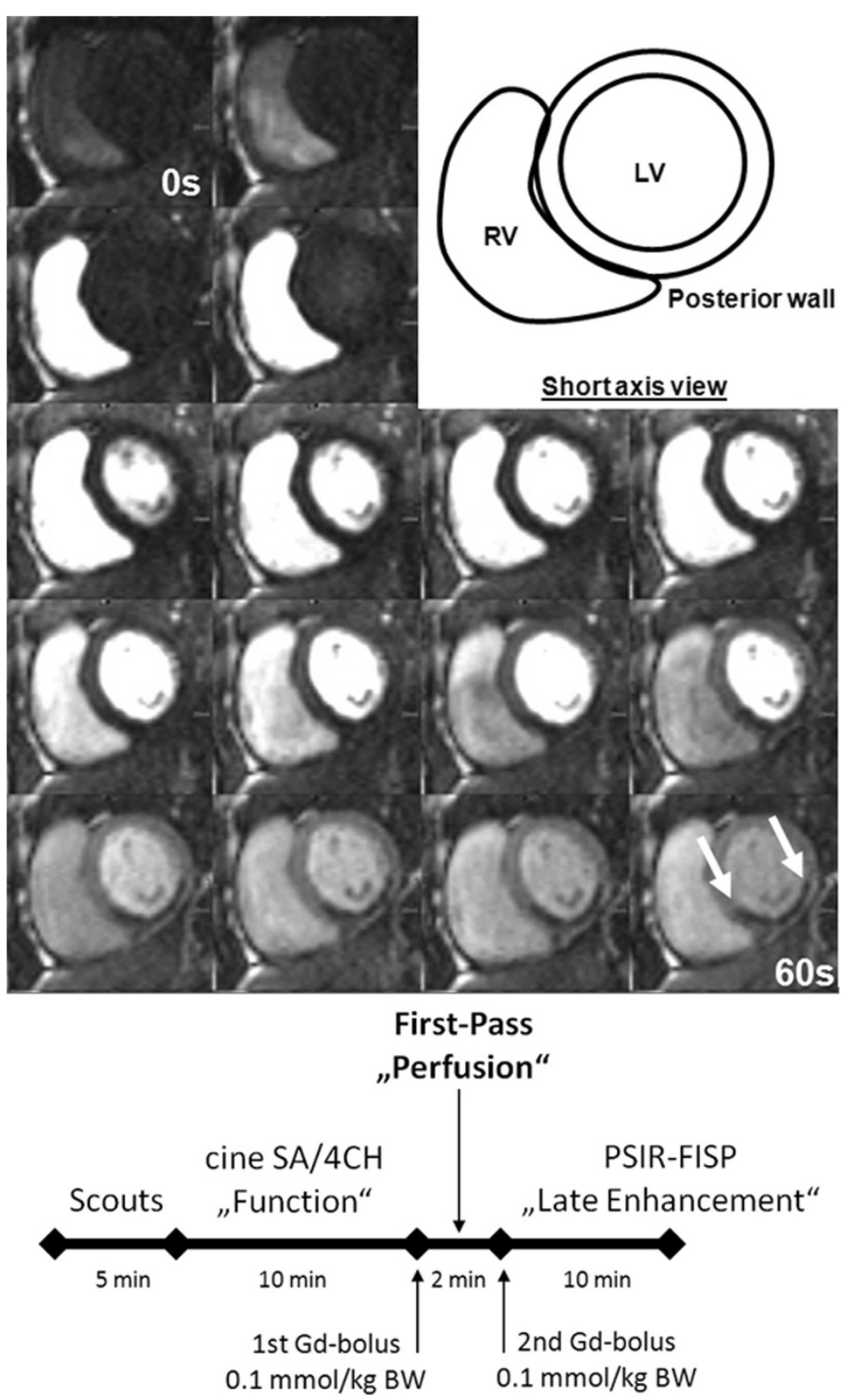

Figure 1 Consecutive slices acquired during first gd-pass (a). The flow-chart below (b) shows the timeline of one CMR. RV: right ventricle, LV: left ventricle, SA: short axis, 4CH: four chamber, Gd: gadolinium, BW: bodyweight, PSIR-FISP: phase-sensitive inversion-recovery fast-imaging-in-steadystate-precession.

two times by an experienced observer (A.M.) blinded to the clinical measurements. Early MVO was considered qualitatively to be present if a region of hypoperfusion persisted on at least 5 consecutive temporal images after contrast bolus arrival in the left ventricle and was located in the subendocardial layer of the infarct core in at least 1 of the short-axis slices. To verify that a true perfusion deficit persisted after passage of the contrast agent, all acquired phases were evaluated [14] (see Figure $1 b)$.
Ten min after a second intravenous gadolinium bolus injection of $0.1 \mathrm{mmol} / \mathrm{kg}$ body mass, LGE-CMR images were acquired by using an ECG-triggered phasesensitive inversion-recovery (PSIR) single shot true-FISP sequence with consecutive short-axis slices as described in detail previously [12]. The area of LGE was evaluated quantitatively for each slice using a commercially available software tool (J-Vision vs. 3.3.16, TIANI Medgraph, Brunn am Gebirge, Austria); enhancement was defined using a threshold of $+5 \mathrm{SD}$ above the signal intensity of 
normal myocardium in the opposite myocardial segment [32-34]. Furthermore, on LGE images a persisting area of low signal, surrounded by enhanced myocardial tissue was considered as late-MVO and quantified by manual contouring of the unenhanced myocardium. Late-MVO mass as well as the percentage of late-MVO myocardium were calculated.

\section{Statistical analysis}

For statistical analysis, the statistical software package SPSS 15.0 (SPSS, Chicago, IL) was used. All results for continuous variables are expressed as mean \pm standard deviation (SD) or, if stated otherwise, as medians with corresponding interquartile range (IQR). KolmogorovSmirnov test was used to test for normal distribution (ND). $\chi^{2}$-test was used to compare categorical variables between groups. ANOVA with Bonferroni post-hoc testing (if ND) and Mann-Whitney $U$ or Kruskal-Wallis test (M-W or K-W, if not ND) were used to determine differences in continuous variables between groups. Pearson test was used for calculation of linear correlations for selected variables if they were ND. Otherwise, Spearman rank correlations were calculated. A p value $\mathrm{p}<0.05$ was considered to indicate statistical significance. Kaplan-Meier survival curves were determined and log-rank comparison was used between groups. Uniand multivariate Cox-regression analysis was used to estimate hazard ratios (HR).

\section{Results}

\section{Patients and follow-up}

Out of 129 patients, 5 patients (3.9\%) did not complete the CMR due to claustrophobia, 124 patients underwent CMR scan within a median of 2 days (IQR: 2-4). Three (2.4\%) scans were not diagnostic because of image quality. The remaining 121 patients were contacted after a median of 1613 days (IQR: 1374-1832 days). Onehundred and seven patients (88.4\%) completed the follow-up. 11 (9.1\%) patients were lost to follow-up, $3(2.5 \%)$ patients could not be contacted for language reasons (see study flow-chart, Figure 2).

\section{Study population baseline characteristics}

The mean age of the study population was $57.0 \pm 12.0$ years (range 31 to 85 years); 99 patients (83.9\%) were male, $19(16.1 \%)$ were female. All patients underwent successful PCI of the culprit lesion with a median delay of 210 minutes (IQR: 120-330). 12 of them obtained additional prehospital fibrinolysis. For detailed patients characteristics, see Table 1.

On initial CMR scans, 74 patients showed early MVO (eMVO+, 69.2\%). eMVO + patients had a higher prevalence of hypertension (65.8\% vs. $\left.45.5 \%, \chi^{2}: 3.88, \mathrm{p}<0.05\right)$ but did not differ from patients without early $\mathrm{MVO}$
(eMVO-) with regard to age, sex, body mass index (BMI) or the prevalence of the remaining cardiovascular risk factors (all $\mathrm{p}>0.05$ ). Furthermore, pain-to-balloon time, administration of prehospital lysis and mean preor post-interventional TIMI flow did not differ significantly between the groups (all p >0.05).

With regard to measures of infarct size, eMVO+ patients had larger infarcts as determined by LE-CMR $(24.9 \pm 15.2$ g vs. $15.5 \pm 16.1$ g, ANOVA $\mathrm{p}<0.01)$, more transmural infarctions $\left(92.9 \%\right.$ vs $66.7 \%, \chi^{2}: 15.27$, $\mathrm{p}<0.002)$ and higher maximum biomarker levels (CKmax: $2844.8 \pm 1992.7$ vs. eMVO- $1492.4 \pm 1802.5 \mathrm{U} / \mathrm{l}$, cTnTmax: $8.02 \pm 5.19$ vs. eMVO- $4.30 \pm 4.71 \mu \mathrm{g} / \mathrm{ml}$, ANOVA p < 0.002) (see Figure 3). Left ventricular ejection fraction was impaired in $\mathrm{eMVO}+$ patients $(39.2 \pm 11.4 \%$ vs eMVO- $46.1 \pm 8.9 \%$, ANOVA $\mathrm{p}<0.01)$ We observed no significant difference in the occurrence of ST-segment resolution $>50 \%(\mathrm{p}>0.05)$.

\section{Primary endpoint}

Sixty-three predefined primary endpoints occurred during follow-up. Primary endpoint occurred in $66.2 \%$ of patients with $\mathrm{MVO}$ and in $42.4 \%$ of patients without MVO $\left(\chi^{2}: 5.34, \mathrm{p}<0.05\right)$. Kaplan-Meier curves demonstrate a significantly reduced event-free survival times for eMVO + patients (median: 930, IQR: 290-1428 days) compared to eMVO- patients (median: 1141, IQR: 3431427 days) (log-rank $\mathrm{p}<0.05$, Figure 4).

Presence of early MVO showed a strong univariate association with the occurrence of the primary endpoint (HR: 1.88, 95\%-CI: 1.04.-3.40, p = 0.029). Early MVO was associated with the occurrence of the primary endpoint when adjusting for age, ejection fraction and infarct size (HR: 2.06, 95\%-CI 1.04-4.09, $\mathrm{p}=0.039$ ). Compared to age above median ( $=57$ years), EF below median $(=40.6 \%)$ and infarct size above median (=19.77 g) eMVO was the strongest independent predictor for the occurrence of the primary endpoint (HR: 2.20, 95\%-CI 1.11-4.36, $\mathrm{p}=0.024$, see Table 2).

\section{Secondary endpoints}

149 individual secondary endpoints occurred in patients with early MVO and 36 in patients without MVO. Table 3 shows the differences between patients with and without MVO regarding the occurrence of secondary endpoints. Patients with MVO suffered more frequently from recurrence of ischemic symptoms (24.7\% vs $6.1 \%$, $\left.X^{2}: 5.13, \mathrm{p}=0.023\right)$, congestive heart failure $(12.3 \% \mathrm{vs}$ $\left.0.0 \%, X^{2}: 4.45, \mathrm{p} 0.035\right)$ and hospitalizations $(60.3 \%$ vs. $\left.39.4 \%, X^{2}: 3.99, \mathrm{p}=0.046\right)$. The incidence of death, $\mathrm{CV}$ death and myocardial re-infarction did not reach statistical significance. 


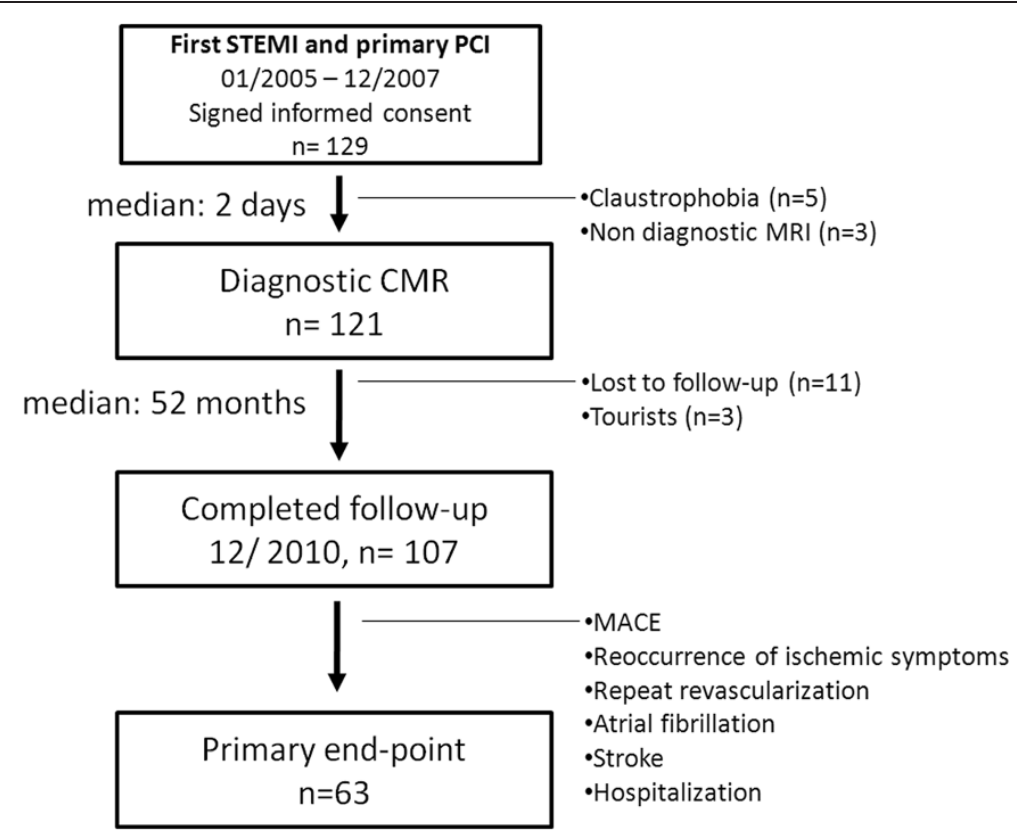

Figure 2 Study flow-chart. STEMI: ST-segment elevation myocardial infarction, PCI: percutaneous coronary interventions, CMR: cardiac magnetic resonance.

\section{Discussion}

The primary results of this study are: (a) patients with early MVO had a higher prevalence of preexisting hypertension, but apart from that, showed similar risk profiles with regard to age, BMI, lipid status, smoking habits, diabetes and family history. (b) In line with most other MVO studies, a strong correlation between infarct size and early MVO was observed. (c) Early MVO is an independent long-term prognosticator of adverse clinical outcomes, especially recurrent ischemic symptoms, heart failure and repeat hospitalization, in a very long follow-up.

\section{Patient characteristics and microvascular obstruction}

$\mathrm{MVO}$ is an increasingly recognised phenomenon after pPCI for STEMI. Prevalence between 25\%[19] and 79\% [20] are described in recent literature. In our study, 69\% of the patients showed eMVO.

Recently, two studies investigated predictors of late MVO defined by CMR, ECG and angiographic analysis $[18,35]$. Their results suggest an association of MVO with age, diabetes [35] and hyperglycemia[18,36]. Due to the sample size of our study we cannot preclude an impact of diabetes or age on the presence of eMVO only by the lack of statistical significance. Our data, however, is in line with a recent larger studie [20]. It is likely that the lower age (56.8 yrs vs. 60 yrs [35], 63 yrs [18] and $66 \mathrm{yrs}[20])$ and the lower prevalence of diabetes (8.4\% vs. $17 \%$ [35], $16 \%$ [18] and $27 \%$ [20]) can be the explanation for the lack of statistical significance in our study group.
In our study, the prevalence of hypertension was significantly higher in patients with eMVO $(65.8 \% v s$. eMVO- 45.5\%) which has, to the best of our knowledge, not been described previously. Data on the effects of hypertension on the cardiac microvasculature suggest impaired endothelial function in hypertensive subjects [37], which in turn might exaggerate microvascular dysfunction in patients after STEMI[1]. Interestingly, Husser et al. observed an inverse association of systolic blood pressure with the presence of MVO, which unfortunately we did not determine. The results of Husser et al. are explained by the authors as a result of a significant higher Killip class in patients with MVO and the resulting cardiogenic shock [35].

There was a trend towards a lower number of active smokers among eMVO + patients $(69.7 \%$ vs. eMVO$50.7 \%, \mathrm{p}=0.052$ ) which was also not observed previously. In analogy with the explanation of Husser et al. that younger patients may lack ischemic preconditioning and coronary collaterals[35], we believe that these mechanisms might explain our results despite the lack of statistical significance.

The presence [20] and extent [13,38] of MVO correlates with measures of infarct size as well as with impaired left ventricular function [20,35]. The ratio of MVO to infarct size not only correlates with biomarker levels [14], but is even of superior prognostic value compared to the extent of MVO alone [38]. Our study is in line with these prior results although the extent of early MVO was not evaluated. Prior results of our study 
Table 1 Patient characteristics

\begin{tabular}{|c|c|c|c|c|}
\hline Clinical Charcteristics & All $(n=107)$ & eMVO + $(n=74)$ & eMVO - $(n=33)$ & p-value \\
\hline Female, $n(\%)$ & $20(18.5)$ & $13(17.6)$ & $7(21.2)$ & .421 \\
\hline Age, years & $56.8 \pm 12.0$ & $57.8 \pm 12.2$ & $54.6 \pm 11.3$ & .217 \\
\hline $\mathrm{BMl}, \mathrm{kg} / \mathrm{m}^{2}$ & $25.5 \pm 4.0$ & $25.8 \pm 2.7$ & $24.9 \pm 5.8$ & .250 \\
\hline Hypertension, n (\%) & $64(59.8)$ & $48(65.8)$ & $15(45.5)$ & $.040^{*}$ \\
\hline Hypercholesterinemia, n(\%) & $87(81.3)$ & $61(83.6)$ & $26(78.8)$ & .367 \\
\hline Smoking, n(\%) & $60(56.1)$ & $37(50.7)$ & $23(69.7)$ & .052 \\
\hline Diabetes mellitus, n(\%) & $9(8.4)$ & 7 (9.6) & $2(6.1)$ & .427 \\
\hline Family history for CVD, n(\%) & $21(19.6)$ & $14(19.2)$ & $7(21.2)$ & .500 \\
\hline Pain-to-balloon, min [median] & 210 (IQR: 120-330) & 210 (IQR: 150-338) & 180 (IQR: 60-270) & .553 \\
\hline Prehospital lysis, n (\%) & $12(11.1)$ & $7(9.5)$ & $4(12.1)$ & .456 \\
\hline TIMI pre $\mathrm{PCl}, \mathrm{n}(\%)$ & & & & .471 \\
\hline MEAN & & $0.38 \pm 0.8$ & $0.58 \pm 0.8$ & .292 \\
\hline 0 & $61(72.6)$ & $46(76.7)$ & $15(62.5)$ & \\
\hline 1 & $10(11.9)$ & $6(10.0)$ & $4(16.7)$ & \\
\hline 2 & $12(14.3)$ & $7(11.7)$ & $5(20.8)$ & \\
\hline 3 & $1(1.2)$ & $1(1.7)$ & $0(0)$ & \\
\hline \multicolumn{5}{|l|}{ TIMI post $\mathrm{PCl}, \mathrm{n}(\%)$} \\
\hline MEAN & & $2.95 \pm 0.4$ & $2.84 \pm 0.5$ & .260 \\
\hline 0 & $1(1.2)$ & $1(0)$ & $0(0)$ & \\
\hline 1 & $1(1.2)$ & $0(0)$ & $1(4.0)$ & \\
\hline 2 & $2(2.3)$ & $0(0)$ & $2(8.0)$ & \\
\hline 3 & $82(95.3)$ & $60(98.4)$ & $22(88.0)$ & \\
\hline CKmax, U/l & $2460.3 \pm 2026.7$ & $2844.8 \pm 1992.7$ & $1492.4 \pm 1802.5$ & $.002^{* *}$ \\
\hline cTnTmax, $\mu \mathrm{g} / \mathrm{ml}$ & $6.96 \pm 5.31$ & $8.02 \pm 5.19$ & $4.30 \pm 4.71$ & $.001^{* *}$ \\
\hline ST-Resolution, n(\%) & $52(80)$ & $33(78.6)$ & $22(81.8)$ & .517 \\
\hline Infarct size, g & $22.0 \pm 16.0$ & $24.9 \pm 15.2$ & $15.5 \pm 16.1$ & $.006^{* *}$ \\
\hline Infarct transmurality, n(\%) & & & & $.002^{* *}$ \\
\hline MEAN & & $98.2 \pm 6.5$ & $85 \pm 25.1$ & $<.001^{* *}$ \\
\hline $0-25$ & $3(3.0)$ & 0 & $3(10.0)$ & \\
\hline $25-50$ & $2(2.0)$ & 0 & $2(6.7)$ & \\
\hline $50-75$ & $10(10.0)$ & $5(7.1)$ & $5(16.7)$ & \\
\hline $75-100$ & $85(85.0)$ & $65(92.9)$ & $20(66.7)$ & \\
\hline Ejection fraction, \% & $41.1 \pm 11.2$ & $39.2 \pm 11.4$ & $46.1 \pm 8.9$ & $.005^{* *}$ \\
\hline Early MVO, n(\%) & $74(69.2)$ & & & \\
\hline Late MVO, n(\%) & $63(58.9)$ & $59(79.7)$ & $4(12.1)$ & $<.001^{* *}$ \\
\hline \multicolumn{5}{|l|}{ NYHA class at follow up, n(\%) } \\
\hline MEAN & & $1.5 \pm 0.8$ & $1.2 \pm 0.5$ & .072 \\
\hline I & $73(73.0)$ & $46(67.6)$ & $26(83.9)$ & \\
\hline$\|$ & $16(16.0)$ & $12(17.6)$ & $4(12.9)$ & \\
\hline III & $9(9.0)$ & $8(11.7)$ & $1(3.2)$ & \\
\hline IV & $2(2.0)$ & $2(2.9)$ & $0(0)$ & \\
\hline
\end{tabular}


Table 1 Patient characteristics (Continued)

\begin{tabular}{llll}
\hline CCS class at follow-up, $\mathrm{n}(\%)$ & & $0.39 \pm 0.7$ \\
MEAN & & $0.43 \pm 0.8$ & $22(71.0)$ \\
0 & $71(71.0)$ & $48(70.6)$ & $6(19.4)$ \\
I & $19(19.0)$ & $13(19.1)$ & $3(9.7)$ \\
II & $9(9.0)$ & $6(8.8)$ & $0(0)$ \\
III & $0(0.0)$ & $0(0)$ & $0(0)$ \\
IV & $1(1.0)$ & $1(1.4)$ & 808 \\
\hline
\end{tabular}

The table is showing the clinical characteristics of the whole study cohort and patients with or without MVO. P-values are given between patients with and patients without MVO $\left({ }^{*} \mathrm{p}<0.05,{ }^{* *} \mathrm{p}<0.01\right)$. Early/late MVO: early microvascular obstruction, BMI: body mass index, CVD: cardiovascular disease, TIMI: thrombolysis in myocardial infarction, PCl: percutaneous coronary intervention, $\mathrm{CK}_{\text {max: }}$ maximum creatine kinase, $\mathrm{CTnT}_{\max }$ : maximum cardiac Troponin T, NYHA: New York Heart Association, CCS: Canadian Cardiovascular Society.

group suggest an additive effect of MVO on myocardial damage [14] and impaired segmental myocardial function and functional recovery [39].

\section{Clinical outcomes}

These effects of MVO on the cellular and functional level might explain why the prognostic value of early [19] and late[38] MVO has frequently been described as superior to that of infarct size alone. The results of the present study extend prior studies by an observation time of 5 years. One limitation of our study, however, is the relatively small sample size, which necessitates the use of a combined primary endpoint.

The question whether early or late MVO is of primary prognostic interest cannot as yet be answered. While deWaha et al. argue that no hard clinical endpoints occurred in patients with early but no late MVO [20], the use of early MVO might have an advantage due to a higher sensitivity in detecting the same pathophysiological phenomenon $[40,41]$. Moreover in a subgroup of 40 patients we previously observed that, beside infarct transmurality, eMVO is the most important imaging parameter in predicting regional functional recovery [39]. On the other hand the use of 3 short-axis slices might underestimate the presence of MVO compared to the six to eight slices acquired on LGE images.
However, we believe that the clinical significance of different results are marginal, especially because the differences in total event-rates are small [20]. The results of our and other previous studies $[11,19,20]$ suggest a superior prognostic value of MVO compared to that of infarct size and ejection fraction.

Infarct size and left ventricular EF alone have been shown to be significant prognostic parameters after STEMI in large CMR-trials [42]. In our study group, however, the univariate impact of classical prognostic markers was not statistically significant. One explanation might be the small sample size of our study, which only allows for the most important parameter, namely MVO, to reach a significant number of clinical endpoints.

In our study, eMVO + patients differed from eMVOpatients in the occurrence of recurrent ischemic symptoms $(24.7 \%$ vs. $6.1 \%, \mathrm{p}<0.05)$, congestive heart failure $(12.3 \%$ vs. $0 \%, \mathrm{p}<0.05)$ and hospitalisations of any kind (60.3\% vs. $39.4 \%, \mathrm{p}<0.05)$. In line with these results, we observed a trend for a higher NYHA class at follow-up (eMVO+: $1.5 \pm 0.8$ vs. eMVO-: $1.2 \pm 0.5, \mathrm{M}-\mathrm{W} \mathrm{p}=0.072$ ) as well as a trend for a higher number of cardiovascular hospitalisations $\left(46.6 \%\right.$ vs. $\left.28.1 \%, \chi^{2}: 3.13, \mathrm{p}=0.077\right)$ and atrial fibrillation $\left(8.2 \%\right.$ vs. $\left.0 \%, \chi^{2}: 2.88, \mathrm{p}=0.09\right)$.

The fact that the presence of MVO leads to a higher rate of recurrent ischemic symptoms during follow-up
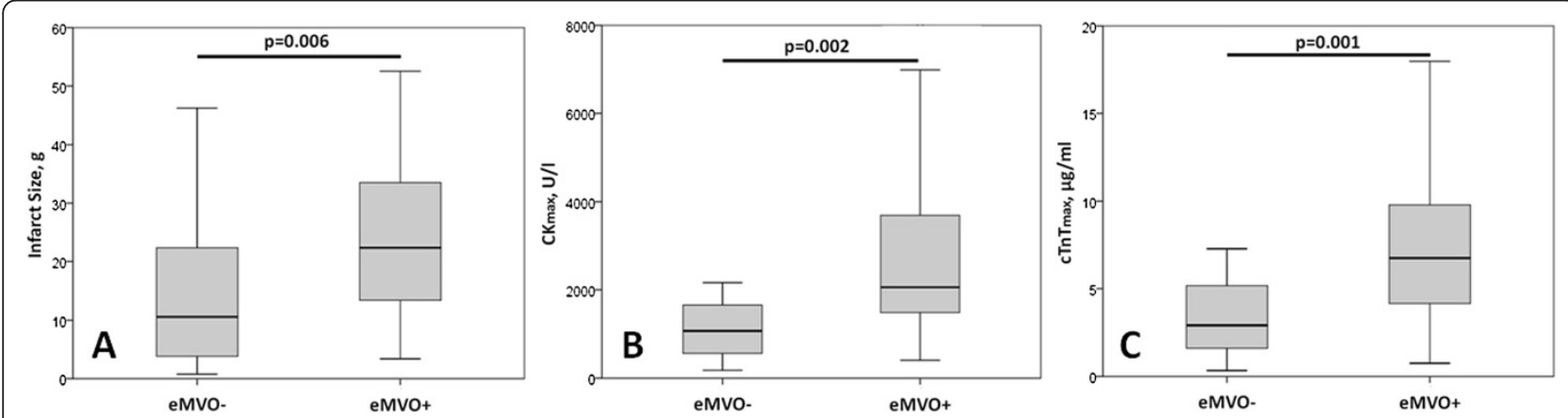

Figure 3 Measures of infarct size in patients with (eMVO+) or without (eMVO-) microvascular obstruction. Boxes give the IQR and whiskers give the 1.5-fold IQR. CK $\max$ : maximum creatine kinase, $\mathrm{CTnT}_{\max }$ : maximum cardiac Troponin T. 


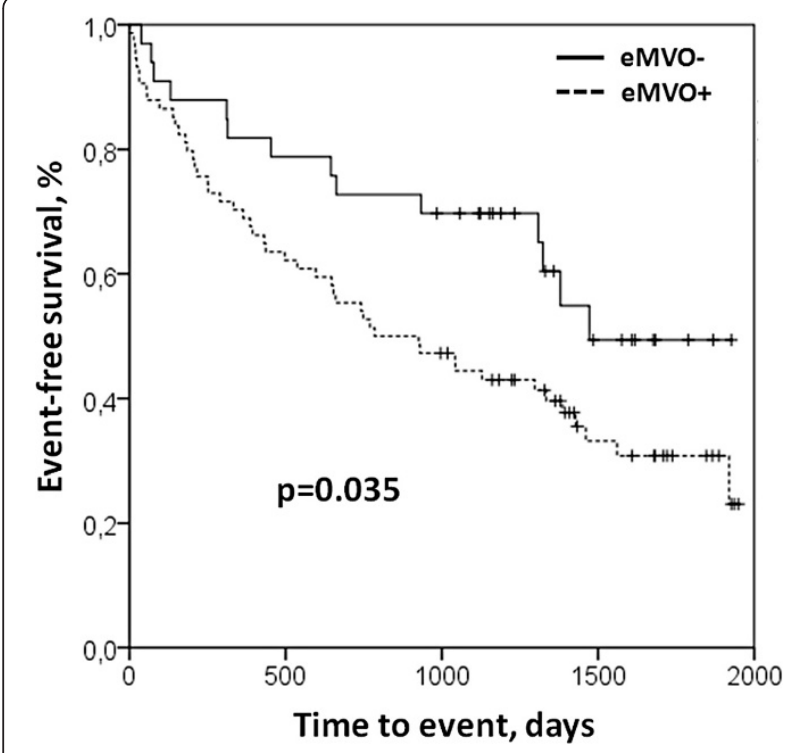

Figure 4 Kaplan-Meier curves showing the differences in eventfree survival between patients without early MVO (eMVO-) and patients with early MVO (MVO-). MVO: microvascular obstruction.

has not been observed previously. One explanation might be a lower number of coronary collaterals in patients with MVO [43]. A second explanation might be found on the level of the microvasculature itself. An impaired endothelium-mediated vasodilation or microvascular spasms during the acute event [44,45], might represent a microvasculature prone to dysfunction, resulting in a higher number of recurrent ischemic symptoms $[46,47]$ and finally MACE $[20]$.

Early re-admission rates for any cause have been proposed as a quality standard after myocardial infarction and represent not only comorbidities but also cardiovascular disease extent. In general STEMI populations $\sim 10 \%$ readmission rates have been reported [48]. In our study 3 patients were re-admitted within 30 days $(2.8 \%)$. All of the admissons were of acute cardiovascular nature and occurred in patients with eMVO. The higher number of long-term admission rates in $\mathrm{eMVO}$ + patients might represent a higher general and cardiovascular

Table 2 Multivariate Cox regression analysis

\begin{tabular}{llll}
\hline & \multicolumn{2}{c}{ Primary Endpoint $(\mathbf{n}=\mathbf{6 3})$} & p-value \\
\cline { 2 - 3 } & $\mathbf{H R}$ & $\mathbf{9 5 \% - C l}$ & \\
\hline eMVO+ & 2.20 & $(1.11-4.36)$ & 0.02 \\
Age $>$ 57 years & 0.96 & $(0.57-1.62)$ & 0.87 \\
EF $<40.6 \%$ & 1.16 & $(0.67-1.99)$ & 0.60 \\
Infarct size $>19.77 \mathrm{~g}$ & 0.69 & $(0.39-1.21)$ & 0.20 \\
\hline
\end{tabular}

Multivariate Cox regression analysis for the occurrence of the primary endpoint. eMVO+: presence of early microvascular obstruction, EF: ejection fraction, HR: hazard ratio, $\mathrm{Cl}$ : confidence interval.
Table 3 Occurrence of secondary endpoints

\begin{tabular}{|c|c|c|c|}
\hline $\begin{array}{l}\text { Secondary Endpoints, } \\
\text { n (\%) }\end{array}$ & $\mathrm{eMVO}+(\mathrm{n}=74)$ & eMVO - $(n=33)$ & p-value \\
\hline Death & $3(4.1)$ & $1(3.0)$ & .797 \\
\hline CV-death & $2(2.7)$ & $1(3.0)$ & .678 \\
\hline Re-infarction & $9(12.3)$ & $2(6.1)$ & .327 \\
\hline Re-CAG & $23(31.5)$ & $8(24.2)$ & .446 \\
\hline Ischemic symptoms & $18(24.7)$ & $2(6.1)$ & $.023^{*}$ \\
\hline Stroke & $1(1.4)$ & $0(0.0)$ & .496 \\
\hline Atrial fibrillation & $6(8.2)$ & $0(0.0)$ & .090 \\
\hline Congestive heart failure & $9(12.3)$ & $0(0.0)$ & $.035^{*}$ \\
\hline Hospitalisation & $44(60.3)$ & $13(39.4)$ & $.046^{*}$ \\
\hline CV-hospitalisation & $34(46.6)$ & $9(28.1)$ & .077 \\
\hline SUM & $49(66.2)$ & $14(42.4)$ & $.021 *$ \\
\hline${ }^{*}$ Chi-square $p<0.05$ & & & \\
\hline
\end{tabular}

morbidity of eMVO + patients, beside yet evaluated cardiovascular factors[35].

In the OPTIMAAL trial it has been shown that atrial fibrillation occurs in up to 6-7\% of patients within 3 years after first STEMI [49]. Further the presence of atrial fibrillation after STEMI is associated with worsening prognosis [50]. To us it seems that atrial fibrillation after STEMI is an important end-point, not only because of prognostic reasons but also because of pathophysiological considerations [51]. Factors associated with atrial fibrillation after STEMI are age, male gender, history of angina, higher Killip classes, higher diastolic blood pressure, and higher pulse rate during the randomization [49]. Some of these factors have also been shown to correlate with infarct size or the presence of MVO [35].

We speculated that adverse remodeling [52] aggravated by the presence of MVO is responsible for our observations. In conclusion, we believe that in our study population of relatively young (56.8 years) patients, the presence of early MVO leads to a higher cardiovascular morbidity after STEMI during a long term follow-up.

\section{Conclusion}

Early MVO, as assessed by first-pass CMR is an independent long-term prognosticator for cardiovascular morbidity after STEMI treated with pPCI. It is associated with a higher prevalence of hypertension and larger infarct size. Our results underline the need for future pre- and post-interventional therapies to avoid early MVO in acute STEMI.

\section{Abbreviations}

AMl: Acute myocardial infarction; BMI: Body mass index; CAG: Coronary angiography; CCS: Canadian cardiovascular society; CK max: maximum creatine kinase CK; Cl: Confidence interval; (LGE-)CMR: (late-Gd-enhanced) Cardiac 
magnetic resonance; $C T_{n} T_{\text {max }}$ : Maximum cardiac Troponin T; CV (D): Cardiovascular (disease); EDV: End-diastolic volume; ESV: End-systolic volume; (LV)EF: (left ventricular) Ejection fraction; PSIR-FISP: Phase-sensitive inversion-recovery fast-imaging-in-steady-state precession; IQR: Interquartile range; (LVMM: (left ventricular) Myocardial mass; (e/l) MVO: (early/late) Microvascular obstruction; ND: Normally distributed; NYHA: New York heart association; pPCl: Primary percutaneous coronary interventions; ROC: Receiver operator characteristics; SD: Standard deviation; SV: Stroke volume; STEMI: ST-segment elevation myocardial infarction; TIMI: Thrombolysis in myocardial infarction.

\section{Competing interest}

The authors declare that they have no competing interest.

\section{Acknowledgements}

We thank Rajam Csordas-lyer for critical reading and helpful comments.

\section{Author details}

${ }^{1}$ Cardiology, University Clinic of Internal Medicine III, Medical University Innsbruck, Innsbruck, Austria. ${ }^{2}$ Department of Radiology I, Medical University Innsbruck, Innsbruck, Austria.

\section{Authors contibution}

KG: Designed study. Collected, analysed and interpreted data. Statistical analysis. Wrote article. MA: Collected, analysed and interpreted CMR data. SS: Collected clinical data. ER: Collected CMR data. SM: Designed study. Statistical analysis. NM: Collected CMR data. JW: Revised the manuscript critically for intellectual content. PO: Revised the manuscript critically for intellectual content. MB: Study Coordinator. Designed study. Revised the manuscript critically for intellectual content. All authors read and approved the final manuscript.

Received: 27 February 2012 Accepted: 12 July 2012

Published: 12 July 2012

\section{References}

1. Jaffe R, Charron T, Puley G, Dick A, Strauss BH: Microvascular obstruction and the no-reflow phenomenon after percutaneous coronary intervention. Circulation 2008, 117:3152-6.

2. Kloner RA, Ganote CE, Jennings RB: The "no-reflow" phenomenon after temporary coronary occlusion in the dog. J Clin Invest 1974, 54:1496-508.

3. Braunwald E, Kloner RA: Myocardial reperfusion: a double-edged sword? J Clin Invest 1985, 76:1713-9.

4. Gibson CM, Ryan KA, Murphy SA, Mesley R, Marble SJ, Giugliano RP, et al.: Impaired coronary blood flow in nonculprit arteries in the setting of acute myocardial infarction. The TIMI Study Group. Thrombolysis in myocardial infarction. J Am Coll Cardiol 1999, 34:974-82.

5. Roe MT, Ohman EM, Maas AC, Christenson RH, Mahaffey KW, Granger CB, et al.: Shifting the open-artery hypothesis downstream: the quest for optimal reperfusion. J Am Coll Cardiol 2001, 37:9-18.

6. Gibson CM, Cannon CP, Murphy SA, Ryan KA, Mesley R, Marble SJ, et al.: Relationship of TIMI myocardial perfusion grade to mortality after administration of thrombolytic drugs. Circulation 2000, 101:125-30.

7. van t Hof AW, Liem A, Suryapranata H, Hoorntje JC, de Boer MJ, Zijlstra F: Angiographic assessment of myocardial reperfusion in patients treated with primary angioplasty for acute myocardial infarction: myocardial blush grade. Zwolle Myocardial Infarction Study Group. Circulation 1998, 97:2302-6.

8. Gibson CM, Murphy SA, Morrow DA, Aroesty JM, Gibbons RJ, Gourlay SG, et al.: Angiographic perfusion score: an angiographic variable that integrates both epicardial and tissue level perfusion before and after facilitated percutaneous coronary intervention in acute myocardial infarction. Am Heart J. 2004, 148:336-40.

9. Lima JA, Judd RM, Bazille A, Schulman SP, Atalar E, Zerhouni EA: Regional heterogeneity of human myocardial infarcts demonstrated by contrastenhanced MRI. Potential mechanisms. Circulation. 1995, 92:1117-25.

10. Judd RM, Lugo-Olivieri CH, Arai M, Kondo T, Croisille P, Lima JA, et al.: Physiological basis of myocardial contrast enhancement in fast magnetic resonance images of 2-day-old reperfused canine infarcts. Circulation 1995, 92:1902-10.
11. Hombach V, Grebe O, Merkle N, Waldenmaier S, Hoher M, Kochs M, et al.: Sequelae of acute myocardial infarction regarding cardiac structure and function and their prognostic significance as assessed by magnetic resonance imaging. Eur Heart J. 2005, 26:549-57.

12. Klug G, Trieb T, Schocke M, Nocker M, Skalla E, Mayr A, et al:: Quantification of regional functional improvement of infarcted myocardium after primary PTCA by contrast-enhanced magnetic resonance imaging. J Magn Reson Imaging 2009, 29:298-304.

13. Trieb T, Mayr A, Klug G, Runge A, Pedarnig K, Pachinger O, et al.: Patterns of myocardial perfusion in the acute and chronic stage after myocardial infarction: A cardiac magnetic resonance study. Eur J Radiol 2012, 81:767-72.

14. Mayr A, Klug G, Schocke M, Trieb T, Mair J, Pedarnig K, et al.: Late microvascular obstruction after acute myocardial infarction: Relation with cardiac and inflammatory markers. Int J Cardiol 2012, 157:391-6.

15. Porto I, Burzotta F, Brancati M, Trani C, Lombardo A, Romagnoli E, et al.: Relation of myocardial blush grade to microvascular perfusion and myocardial infarct size after primary or rescue percutaneous coronary intervention. Am J Cardiol 2007, 99:1671-3.

16. Wu KC, Kim RJ, Bluemke DA, Rochitte CE, Zerhouni EA, Becker LC, et al.: Quantification and time course of microvascular obstruction by contrastenhanced echocardiography and magnetic resonance imaging following acute myocardial infarction and reperfusion. J Am Coll Cardiol 1998, 32:1756-64.

17. Yip HK, Chen MC, Chang HW, Hang CL, Hsieh YK, Fang CY, et al.: Angiographic morphologic features of infarct-related arteries and timely reperfusion in acute myocardial infarction: predictors of slow-flow and no-reflow phenomenon. Chest 2002, 122:1322-32.

18. Niccoli G, Spaziani C, Cosentino N, Lombardo A, Fracassi F, Cataneo L, et al.: Predictors of myocardial microvascular obstruction in patients treated by primary percutaneous coronary intervention and a short ischemic time. Int J Cardiol 2011, 153:113-5.

19. Wu KC, Zerhouni EA, Judd RM, Lugo-Olivieri CH, Barouch LA, Schulman SP, et al.: Prognostic significance of microvascular obstruction by magnetic resonance imaging in patients with acute myocardial infarction. Circulation 1998, 97:765-72.

20. de Waha S, Desch S, Eitel I, Fuernau G, Zachrau J, Leuschner A, et al.: Impact of early vs. late microvascular obstruction assessed by magnetic resonance imaging on long-term outcome after ST-elevation myocardial infarction: a comparison with traditional prognostic markers. Eur Heart $J$ 2010, 31:2660-8.

21. Kloner RA, Forman MB, Gibbons RJ, Ross AM, Alexander RW, Stone GW: Impact of time to therapy and reperfusion modality on the efficacy of adenosine in acute myocardial infarction: the AMISTAD-2 trial. Eur Heart J. 2006, 27:2400-5.

22. Ross AM, Gibbons RJ, Stone GW, Kloner RA, Alexander RW: A randomized, double-blinded, placebo-controlled multicenter trial of adenosine as an adjunct to reperfusion in the treatment of acute myocardial infarction (AMISTAD-II). J Am Coll Cardiol 2005, 45:1775-80.

23. Thiele H, Schindler K, Friedenberger J, Eitel I, Furnau G, Grebe E, et al.: Intracoronary compared with intravenous bolus abciximab application in patients with ST-elevation myocardial infarction undergoing primary percutaneous coronary intervention: the randomized Leipzig immediate percutaneous coronary intervention abciximab IV versus IC in ST-elevation myocardial infarction trial. Circulation 2008, 118:49-57.

24. De Luca G, Dudek D, Sardella G, Marino P, Chevalier B, Zijlstra F: Adjunctive manual thrombectomy improves myocardial perfusion and mortality in patients undergoing primary percutaneous coronary intervention for ST-elevation myocardial infarction: a meta-analysis of randomized trials. Eur Heart J. 2008, 29:3002-10.

25. Jaffe R, Dick A, Strauss BH: Prevention and treatment of microvascular obstruction-related myocardial injury and coronary no-reflow following percutaneous coronary intervention: a systematic approach. JACC Cardiovasc Interv. 2010, 3:695-704.

26. Gibson CM, Maehara A, Lansky AJ, Wohrle J, Stuckey T, Dave R, et al: Rationale and design of the INFUSE-AMI study: A 2 × 2 factorial, randomized, multicenter, single-blind evaluation of intracoronary abciximab infusion and aspiration thrombectomy in patients undergoing percutaneous coronary intervention for anterior ST-segment elevation myocardial infarction. Am Heart J 2011, 161:478-86. e7.

27. Alpert JS, Thygesen K, Antman E, Bassand JP: Myocardial infarction redefined-a consensus document of The Joint European Society of 
Cardiology/American College of Cardiology Committee for the redefinition of myocardial infarction. J Am Coll Cardiol 2000, 36:959-69.

28. Mayr A, Mair J, Schocke M, Klug G, Pedarnig K, Haubner BJ, et al.: Predictive value of NT-pro BNP after acute myocardial infarction: relation with acute and chronic infarct size and myocardial function. Int J Cardiol 2011, 147:118-23.

29. Matthews JN, Altman DG, Campbell MJ, Royston P: Analysis of serial measurements in medical research. BMJ 1990, 300:230-5.

30. Klug G, Mayr A, Mair J, Schocke M, Nocker M, Trieb T, et al.: Role of biomarkers in assessment of early infarct size after successful p-PCI for STEMI. Clin Res Cardiol 2011, 100:501-10.

31. Klug G, Wolf C, Trieb T, Frick M, Koehler A, Schocke MF, et al.: Evaluation of transient apical ballooning with cardiac magnetic resonance imaging and 31-phosphorous magnetic resonance spectroscopy. Int I Cardiol 2007, 118:249-52.

32. Beek AM, Kuhl HP, Bondarenko O, Twisk JW, Hofman MB, van Dockum WG, et al.: Delayed contrast-enhanced magnetic resonance imaging for the prediction of regional functional improvement after acute myocardial infarction. J Am Coll Cardiol 2003, 42:895-901.

33. Bondarenko O, Beek AM, Hofman MB, Kuhl HP, Twisk JW, van Dockum WG, et al.: Standardizing the definition of hyperenhancement in the quantitative assessment of infarct size and myocardial viability using delayed contrast-enhanced CMR. J Cardiovasc Magn Reson 2005, 7:481-5.

34. Nowosielski M, Schocke M, Mayr A, Pedarnig K, Klug G, Kohler A, et al.: Comparison of wall thickening and ejection fraction by cardiovascular magnetic resonance and echocardiography in acute myocardial infarction. J Cardiovasc Magn Reson 2009, 11:22.

35. Husser O, Bodi V, Sanchis J, Nunez J, Lopez-Lereu MP, Monmeneu JV, et al.. Predictors of cardiovascular magnetic resonance-derived microvascular obstruction on patient admission in STEMI. Int J Cardiol 2011, Oct 2011. Epub ahead of print.

36. Jensen CJ, Eberle HC, Nassenstein K, Schlosser T, Farazandeh M, Naber CK, et al:: Impact of hyperglycemia at admission in patients with acute ST-segment elevation myocardial infarction as assessed by contrast-enhanced MRI. Clin Res Cardiol 2011, 100:649-59.

37. Panza JA, Quyyumi AA, Brush JE Jr, Epstein SE: Abnormal endotheliumdependent vascular relaxation in patients with essential hypertension. N Engl J Med 1990, 323:22-7.

38. de Waha S, Desch S, Eitel I, Fuernau G, Lurz P, Leuschner A, et al.: Relationship and prognostic value of microvascular obstruction and infarct size in ST-elevation myocardial infarction as visualized by magnetic resonance imaging. Clin Res Cardiol 2012, 101 6:487-495.

39. Mayr A, Pedarnig K, Klug G, Schocke M, Pachinger O, Jaschke W, et al.: Regional functional recovery after acute myocardial infarction: a cardiac magnetic resonance long-term study. Int I Cardiovasc Imaging. 2011, Oct 1 Epub ahead of print.

40. Kim RJ, Chen EL, Lima JA, Judd RM: Myocardial Gd-DTPA kinetics determine MRI contrast enhancement and reflect the extent and severity of myocardial injury after acute reperfused infarction. Circulation 1996, 94:3318-26.

41. Mather AN, Lockie T, Nagel E, Marber M, Perera D, Redwood S, et al.: Appearance of microvascular obstruction on high resolution first-pass perfusion, early and late gadolinium enhancement CMR in patients with acute myocardial infarction. J Cardiovasc Magn Reson 2009, 11:33.

42. Klem I, Shah DJ, White RD, Pennell DJ, van Rossum AC, Regenfus M, et al.: Prognostic value of routine cardiac magnetic resonance assessment of left ventricular ejection fraction and myocardial damage: an international, multicenter study. Circ Cardiovasc Imaging. 2011, 4:610-9.

43. Desch S, Eitel I, Schmitt J, Sareban M, Fuernau G, Schuler G, et al.: Effect of coronary collaterals on microvascular obstruction as assessed by magnetic resonance imaging in patients with acute ST-elevation myocardial infarction treated by primary coronary intervention. Am J Cardiol 2009, 104:1204-9.

44. Sun H, Mohri M, Shimokawa H, Usui M, Urakami L, Takeshita A: Coronary microvascular spasm causes myocardial ischemia in patients with vasospastic angina. J Am Coll Cardiol 2002, 39:847-51.

45. Pries AR, Habazettl H, Ambrosio G, Hansen PR, Kaski JC, Schachinger V, et al.: A review of methods for assessment of coronary microvascular disease in both clinical and experimental settings. Cardiovasc Res 2008, 80:165-74.

46. Sambuceti G, Marzilli M, Fedele S, Marini C, L'Abbate A: Paradoxical increase in microvascular resistance during tachycardia downstream from a severe stenosis in patients with coronary artery disease: reversal by angioplasty. Circulation 2001, 103:2352-60.

47. Marzilli M, Sambuceti G, Fedele S, L'Abbate A: Coronary microcirculatory vasoconstriction during ischemia in patients with unstable angina. J Am Coll Cardiol 2000, 35:327-34.

48. Kociol RD, Lopes RD, Clare R, Thomas L, Mehta RH, Kaul P, et al: International variation in and factors associated with hospital readmission after myocardial infarction. JAMA. 2012, 307:66-74.

49. Lehto M, Snapinn S, Dickstein K, Swedberg K, Nieminen MS: Prognostic risk of atrial fibrillation in acute myocardial infarction complicated by left ventricular dysfunction: the OPTIMAAL experience. Eur Heart J. 2005, 26:350-6.

50. Schmitt J, Duray G, Gersh BJ, Hohnloser SH: Atrial fibrillation in acute myocardial infarction: a systematic review of the incidence, clinical features and prognostic implications. Eur Heart J. 2009, 30:1038-45.

51. Aronson D, Mutlak D, Bahouth F, Bishara R, Hammerman H, Lessick J, et al.: Restrictive left ventricular filling pattern and risk of new-onset atrial fibrillation after acute myocardial infarction. Am J Cardiol 2011, 107:1738-43.

52. Lombardo A, Niccoli G, Natale L, Bernardini A, Cosentino N, Bonomo L, et al.: Impact of microvascular obstruction and infarct size on left ventricular remodeling in reperfused myocardial infarction: a contrastenhanced cardiac magnetic resonance imaging study. Int J Cardiovasc Imaging 2011, 28 4:835-842.

\section{doi:10.1186/1532-429X-14-46}

Cite this article as: Klug et al:: Prognostic value at 5 years of microvascular obstruction after acute myocardial infarction assessed by cardiovascular magnetic resonance. Journal of Cardiovascular Magnetic Resonance 2012 14:46.

\section{Submit your next manuscript to BioMed Central and take full advantage of:}

- Convenient online submission

- Thorough peer review

- No space constraints or color figure charges

- Immediate publication on acceptance

- Inclusion in PubMed, CAS, Scopus and Google Scholar

- Research which is freely available for redistribution
C Biomed Central 